\title{
Heterotrophic bacteria in botte refill stations
}

\author{
Sophie Fantillo ${ }^{1}$, Dr. Helen Heacock ${ }^{2}$ \\ ${ }^{1}$ Lead Author, B. Tech Student, School of Health Sciences, British Columbia Institute of Technology, 3700 \\ Willingdon Ave, Burnaby BC V5G 3H2 \\ ${ }^{2}$ Supervisor, School of Health Sciences, British Columbia Institute of Technology, 3700 Willingdon Ave, Burnaby BC \\ V5G $3 \mathrm{H} 2$
}

\begin{abstract}
Background: Heterotrophic bacteria are commonly found in water supplies where there is inadequate or nonexistent disinfection. Water coolers are known to have high HPC levels due to the filtered, non-chlorinated water provided. Water bottle refill stations utilize a carbon filter which can act as a food source for HPC. This study measured HPC levels in water samples from bottle refill stations to determine whether there is a difference compared to tap water at BCIT.
\end{abstract}

Method: Standard Method 9060 A was used to collect water from bottle refill stations to compare to nonfiltered tap water. Samples were plated using R2A Agar and incubated for 7 days before enumerating HPC from water samples. Samples were collected from specific drinking water fountains that contained Carbon Filters and compared to the nearest tap water source.

Results: Mean HPC levels in bottle refill stations were found at $95 \mathrm{cfu} / \mathrm{mL}$ while mean HPC levels in tap water were $55 \mathrm{cfu} / \mathrm{mL}$. A two-sample T-test confirmed that the mean HPC levels of bottle refill stations and tap water are statistically significantly different $(P=0.000124)$. Although the findings were statistically significant, the study's power was low at $11 \%$.

Conclusion: Based on the results, drinking water obtained from bottle-refill stations at BCIT contained on an average higher level of HPC compared to tap water. Overall, HPC levels were below recommended levels in drinking water and not considered to have any harmful effects. To continue the safe use of bottle refill stations, facilities should develop and follow written procedures to maintain stations and ensure regular changing of filters.

Key words: Heterotrophic, HPC, carbon filter, drinking water

\section{INTRODUCTION}

Water is an essential component for all living things, and identified as a remedy for some ailments. Water is often viewed to benefit one's health. $70 \%$ of Earth's surface is covered in water. Of that, only 3 percent is fresh water for drinking, found in aquifers, ice caps, surface lakes and as water vapor. Water is capable of carrying pathogenic bacteria. Without adequate treatment and disinfection, water from the environment has the potential to cause health consequences when consumed.

Fortunately, in Canada, it is rather rare to see illnesses associated with municipal drinking water due to sophisticated treatment facilities. One of the major components that keeps potable water safe is the addition of chlorine as a disinfectant. Chlorine has the ability to kill or inactivate harmful bacteria.

One type of microbe commonly found in drinking water is heterotrophic bacteria. Heterotrophs feed 
on carbon source, contribute to the make-up of biofilms, and are easily killed by the disinfecting agent chlorine. Despite having no direct health effects, biofilms in water sources are capable of harboring pathogenic bacteria which in turn could be ingested by consumers (Percival, Walker, \& Hunter, 2000c).Heterotrophic bacteria are typically used to evaluate the effectiveness of the residual disinfection in the water distribution system.

The purpose of this literature review is to examine the scientific evidence of heterotrophic bacterial growth in public drinking water. It will identify research, policy and knowledge gaps, which are important to identify as the environmental movement becomes stronger every day.

\section{LITERATURE REVIEW}

This study compared heterotrophic bacteria levels in filtered water provided from water bottle refill stations to non-filtered tap water fountains at BCIT Burnaby Campus. These bottle refill stations provide filtered water or non-filtered water, depending on the model. The stations at BCIT execute a carbon filter to remove chlorine from the drinking water to provide a more desirable taste (Pereira, 2016). Stations that employed carbon filtered was provided by BCIT Facilities Department. Based on previous research, it is known that water coolers found in private homes and other various locations providing non-chlorinated water are found to have high HPC levels (Levesque et al., 1994). This study examined if these bottle refill stations also have elevated Heterotrophic Plate Count (HPC) levels.

The Guidelines for Canadian Drinking Water Quality state that heterotrophic bacteria do not contribute to any adverse health effects (Federal-ProvincialTerritorial Committee on Drinking Water, 2014). Many parameters that have the potential to cause illness are set in the guidelines as far as acceptable levels. The parameters that are typically most focused on are total coliforms and fecal coliforms, which should never exist in drinking water (FederalProvincial-Territorial Committee on Drinking Water, 2014). Heterotrophic Plate Count (HPC) acts as an indicator organism to show there is a problem somewhere within the distribution system.

The issue of bottle-less water dispensers was brought to the attention of the author as the emergence of filtered drinking water bottle refill stations increased from Spring 2016 to Fall 2016 returning to school at British Columbia Institute of Technology after summer vacation. These stations are sought after by students as they provide cooled water with an improved taste in comparison to municipal drinking fountains, along with a handy ergonomic spout for filling water bottles. They also provide savings as students can refrain from purchasing bottled water.

The intent of these bottle stations is to reduce the amount of purchased bottled water and to have a better impact on the environment. Although plastic water bottles can be recycled, the lifetime of recycling plastic is limited. The area of bottled water has always been of interest as it is often considered a waste of money and resources to purchase bottled water especially when Vancouver employs advanced water treatment technology to provide clean, safe, and tasty drinking water (Metro Vancouver, 2016). Water treatment systems are designed to eliminate hazards and to provide safe water to the public. Many people believe that chlorine found in drinking water is harmful and find the taste unenjoyable so they resort to purchasing bottled water. Placing these bottle refill stations in highly populated areas such as universities encourages people to bring a reusable water bottle of their own instead of relying on purchasing bottled water.

\section{Drinking Water Guidelines}

Water from nature is never pure and can pick up anything in which it can come into contact. The Metro Vancouver drinking water source comes from three highly protected watersheds (Metro Vancouver, 2016). The surface water lakes, which have restricted public access, are found in a protected area. This makes the raw water of great quality; however, it still needs treatment to eliminate microorganisms. The Guidelines for Canadian Drinking Water Quality set out Maximum 
Acceptable Concentrations (MAC's) of certain substances in drinking water. Guidelines are designed to protect the health of most vulnerable members of society (Federal-Provincial-Territorial Committee on Drinking Water, 2014). These guidelines deal with microbiological, chemical and radiological contaminants. They also address physical characteristics of water such as taste and odor.

As HPC is not considered to have any health effects, no MAC has been set by the Guidelines for Canadian Drinking Water Quality. The guidelines state that HPC is a useful tool for monitoring general bacteriological water quality throughout the treatment process and in the distribution system (Federal-Provincial-Territorial Committee on Drinking Water, 2014). If HPC is found in water sources after bacteriological testing, the results are not an indicator of water safety and should not be used as an indicator of potential human health effects (Federal-Provincial-Territorial Committee on Drinking Water, 2014).

When comparing to the United States (US), it can be agreed that HPC has no health effects. However, US guidelines state that bacterial counts in drinking water should not exceed 500 colonies $/ \mathrm{mL}$ of water sampled (Epa, 2009). Despite not having any direct adverse effects on health, HPC can harbor some pathogens that in the right conditions could become hazardous to consumers. The presence of HPC in drinking water is used as an indicator of poor maintenance of the distribution system.

Typically, in municipal water sources, HPC isn't of much concern because of the added disinfectant. Potable water from the municipality undergoes extensive treatment before it reaches the public for consumption. Chlorine is added in a controlled concentration as a disinfectant to kill any pathogens and holds a residual for the distribution system to inhibit any regrowth. The disinfection step implies that potable water contains injured or dormant microorganisms since it would be impossible to remove all organisms (Levesque et al., 1994). The degree of injury remains uncertain; microorganisms are thought to be metabolically active but incapable of replication on culture media (Percival, Walker, \& Hunter, 2000a). Once the chlorine residual is removed using a filter, any microbes that survived in the treatment and distribution system are presented with ideal conditions for multiplication.

\section{Biofilms}

Build-up of microorganisms in a cluster is referred to as a biofilm. Biofilms, commonly found in water distribution systems as well as water dispensers such as hoses, fountains and water coolers, are a group of bonded microbiological cells attached to a surface (Percival, Walker, \& Hunter, 2000b). Biofilms can provide a safe-haven for growth of various types of pathogens. Within the distribution system of drinking water, bacteria can adhere to surface walls which gives microorganisms chlorine protection (Percival et al., 2000a). Biofilms are typically small and patchy, however if a large accumulation forms it could corrode the pipeline resulting in positive bacteriological tests (coliforms). For heterotrophic bacteria to grow and multiply in water, various amounts of carbon, nitrogen and phosphorous must be present (Percival et al., 2000b). Microbes grow and multiply after attaching to piping surface in distribution system, consuming nutrients evident within the film.

If no chlorine residual in a drinking water source is present, there is a potential of both heterotrophic and coliform regrowth. Likewise, when chlorine is deliberately removed by the consumer to enhance taste, heterotrophic bacteria have the proper environment to grow if they have survived the treatment process. Carbon filters typically used in private homes are designed to remove chlorine via adsorption. This carbon can also act as a nutrient source for pathogenic bacteria (Percival et al., 2000a).

\section{Bottle Refill Stations}

With the increased environmental impact awareness among the public, an abundance of bottle refill stations providing filtered drinking water have been implemented in various publicly accessible areas (Figure 1). Water stations often have a meter that shows the consumer the number 
of bottled waters that have been saved by using this bottle refill station. These stations have an excellent impact on preventing waste and encouraging people to feel as if they have contributed to a healthier planet.

Water refill stations should be treated in a similar manner that water coolers are treated. Nonchlorinated water passes through a dispenser and into a drinking receptacle. This creates a great environment for biofilms to develop and potentially contaminate the drinking water. With routine cleaning, disinfection, and changing of filters there would be no concern for HPC.

Many advantages of water refill stations can be enjoyed by all consumers such as: improved taste, cooled temperature, cost effectiveness as compared to purchasing bottled water, reduced lead and other metals, avoiding BPA exposure from recyclable plastic bottles, less environmental impact, and reducing water consumption or water waste. Production facilities consume more water to produce a plastic bottle than to fill it. There are few disadvantages to the consumer, however it is the operator or supplier that is faced with some challenges: units are expensive to purchase, filters need to be routinely changed which is another added cost, training and cleaning of the units is required, and there is no direct revenue increase by providing the service ("ELKAY | Elkay EZH2O Bottle Filling Station with Single Cooler Filtered, $8 \mathrm{GPH}$ Vandal-Resistant Stainless LVRC8WSK," n.d.). Consumers are required to carry their own bottle any may see this as a burden.

\section{Health Impacts}

A study in California looked at the microbiological water quality from water vending machines. A water vending machine dispenses "bulk" water the same way a public drinking fountain does but it charges a fee. These vending machines are closely related to bottle refill stations in their structure and availability. The largest difference is these vending machines have a reservoir for cooling water (like water coolers), while bottle refill stations have a condensing unit and no reservoir. As these vending machines provided filtered water via UV light, they are covered by legislation and held to the same standards as bottled water (Schillinger \& S, 2004). The Health Department did not routinely inspect these machines; however, they would respond to any complaints. The vending machines were advertised to be

serviced once a week to be cleaned inside and out and the filter checked and replaced if necessary per some companies. The study found that $48 \%$ of their samples had HPC greater than 500 $\mathrm{cfu} / \mathrm{ml}$, with a mean of $889 \mathrm{cfu} / \mathrm{ml}$ (Schillinger \& $S$, 2004). In this study, many different bacteria were found in these waters including coliforms and fecal coliforms. This shows how the water quality can diminish if dispensers are not properly maintained. Had these machines been cleaned weekly as advertised, the levels found wouldn't have been so high.

Schillinger's study is an example of the potential for other drinking fountains that offer filtered water to the public. Higher levels of HPC are typically found in water coolers when compared to municipal supply due to the lack of disinfectant (Levesque et al., 1994). The need for regular cleaning is apparent and needs to be conducted to ensure water provided to the public is safe. Without adequate cleaning, the public are at higher risk for gastrointestinal illness.

\section{Manufacturer's Instructions}

Carbon filters need to be changed per manufacturer's instructions. For example, Brita point-of-use filters (jug style) recommend being changed every 40 gallons of water or every 2 months (Brita Water Filtration Process, 2014). 
Franke by Oasis which provides commercial standard drinking fountains with bottle refill stations utilizes Versafilter filtration system. It is recommended to change its filter every 1250 gallons or every 5 months (Oasis, n.d.). Elkay EZH2O is another brand that provides commercial standard drinking fountains and bottle refill stations using WaterSentry filter technology. Various styles are offered, but for bottler refill stations it is recommended to change the filter every 3000 gallons. A light sensor alerts consumers when it's time to change the filter ("ELKAY | Elkay EZH2O Bottle Filling Station with Single Cooler Filtered, 8 GPH Vandal-Resistant Stainless LVRC8WSK," n.d.).

\section{Public Health Implications}

Public Health officials need to be aware that these drinking fountains exist in various locations. It is commonly known that private water coolers (sometimes found in public areas as well) need to be regularly cleaned and sanitized to kill any pathogens being harbored in a biofilm (Levesque et al., 1994). The same can be said for public bottle refill stations dispensing filtered water. These bottle refill stations have been designed to limit any contamination, however it is unknown the level of risk they face. If an outbreak were to occur that could be traced to water, water dispensers should be considered when trying to identify the source. Generally, these water stations provide clean and healthy water, however with inconsistent maintenance the risk for contamination still exists.

\section{Gaps in Research}

As commercial bottle refill stations have only been publicly available since 2010, limited academic research has been conducted around this time frame (Elkay, 2017). To the knowledge of the researcher, no existing studies examined water quality from bottle refill stations or the cleanliness of these stations. The only policy surrounding bottle refill stations is that water must meet the Guidelines for Canadian Drinking Water Quality.

\section{Research Question}

The following null $\left(\mathrm{H}_{0}\right)$ and alternate $\left(\mathrm{H}_{\mathrm{a}}\right)$ hypotheses were established to determine levels of HPC in drinking water:

$\mathbf{H}_{0}$ : There is no difference between mean HPC levels in drinking water from filtered bottle refill stations compared to the mean HPC levels in drinking water from tap water fountains at BCIT.

$\mathbf{H}_{\mathrm{a}}$ : There is a difference between mean HPC levels in drinking water from filtered bottle refill stations compared to the mean HPC levels in drinking water from tap water fountains at BCIT.

\section{METHODS AND MATERIALS}

The methods for measuring heterotrophic bacteria were based on the Standard Methods for the Examination of Water and Wastewater (APHA/AWWA/WEF, 2005), however due to budget and time restrictions, a simplified version of the methods was utilized. The following materials were used:

\begin{tabular}{|c|c|}
\hline Description & Quantity \\
\hline - Sterile sample bottles & 38 \\
- with sodium thiosulphate & \\
- Cooler with ice packs & 1 \\
Petri plates with solidified & 38 \\
- R2A agar & 38 \\
- Sterile pipette & 1 \\
\hline
\end{tabular}

\section{Sample Collection}

Drinking water samples were collected using aseptic techniques to eliminate the chances of contamination. The researcher washed her hands prior to sample collection. Water was collected following Standard Methods 9060A (Association, 1999). Sterile bottles containing sodium thiosulphate were used to collect $100 \mathrm{~mL}$ of water for each sample at different locations. Sodium thiosulphate is used as a neutralizing agent for residual chlorine in drinking water and is not required for filtered water samples. To reduce risk of sample contamination, sodium thiosulphate was left in the bottles used for filtered water samples. Sample bottles were labelled with date, location and coded for filtered or non-filtered water prior to collection at each location. In order to correctly 
represent water that would be consumed, samples were taken immediately when water is turned on rather than letting the water flow for 2-3 minutes as recommended (Association, 1999).

Samples were taken at a total of 12 different locations on BCIT Burnaby Campus. Three of the locations listed were not sampled (one was out of order, and the researcher was refused access at the remaining two). Secondary samples were taken at 7 of the locations chosen at random to increase the sample size. A total of 38 samples were taken. The tap water drinking fountain samples were chosen based on closest physical location to the bottle refill station being sampled. Most locations did not have tap water drinking fountain and samples were taken from the tap in the closest women's bathroom. Tap water sources from faucets (opposed to bathrooms) did not have their screen removed as recommended by the Standard Methods as the researcher was looking for a representative of consumed drinking water (Association, 1999). Bottle refill stations had a bottle filler as well as a drinking spout; samples were collected from the bottle filler and not the drinking spout. Similarly, to tap water, screens were not removed from the bottle refill stations. Samples were stored in a cooler containing ice packs during entire collection time then placed into a refrigerator until enumeration could occur. Samples were analyzed within 24 hours of collection as required (Health Canada, 2012).

\section{Bacteriological Analysis}

Each plate was marked with sample number, date and location as per Standard Methods (APHA/AWWA/WEF, 2005). There are a few ways in which HPC can be enumerated including the spread plate method, the pour plate method, and membrane filtration (Health Canada, 2012). Each $100-\mathrm{mL}$ sample was shaken for 15 seconds prior to pipetting to evenly distribute any present bacteria in the sample. A sterile pipette was used to take $1 \mathrm{~mL}$ from each sample and place onto a plate with solidified R2A agar with the pipette being discarded after use. Samples were swirled on the plate until no free liquid could be seen, and left for 10 minutes on a level surface for the media to absorb the water sample. Samples were then transferred and inverted to incubate at $20-28^{\circ} \mathrm{C}$ as per agar instructions (Neogen, 2009). Samples were enumerated 7 days after plating.

Inclusion and Exclusion. Water bottle refill stations on the list provided by Facilities Department at BCIT were included in this study (Pereira, 2016). These stations utilize a carbon filter and have a bottle refill spout. A few of the locations on the list were not bottle refill stations, however smaller fountains that employed a carbon filter. These water sources were categorized as bottle refill stations for the sake of the study due to the same method of filtration. Bottle refill stations that do not utilize a carbon filter were excluded from this study for the "filtered" variable. As far as tap water, all sources on the BCIT Burnaby Campus were considered as part of the included, however only certain sources were selected (demonstrated in methods).

\section{STATISTICAL ANALYSIS}

Description of data. The data being measured is discrete numerical. This type of measurement uses whole numbers only - the researcher counted colonies formed on agar plates. It would be incorrect to say that half a colony was found, it is either there or it is not therefore it is discrete opposed to continuous. The descriptive statistics used include mean, mode, median, range and standard deviation.

Statistical package used. NCSS 11 and Microsoft Excel 2016 were utilized in this study. Microsoft provided information on the descriptive statistics, while NCSS calculated complex inferential statistics required to determine significance of the research. Information on both sample types, referred to "filtered" and "tap" were collected with 19 data points each and imputed into NCSS for analysis.

Descriptive Statistics. The following table summarizes the descriptive statistics recorded by the researcher: 


\begin{tabular}{lrrlrr} 
Mean & 95.8 & 55.7 & Skewness & 1.6 & 3.8 \\
Standard Error & 27.3 & 42.8 & Range & 395.0 & 756.0 \\
Median & 40.0 & 0.0 & Minimum & 2.0 & 0.0 \\
Standard Deviation & 115.9 & 181.5 & Maximum & 397.0 & 756.0 \\
Sample Variance & 13435.5 & 32952.4 & Sum & 1724.0 & 1003.0 \\
Kurtosis & 1.7 & 15.0 & Count & 18.0 & 18.0 \\
\cline { 3 - 5 }
\end{tabular}

Inferential Statistics. The inferential statistics utilized was a two sample T-test. These tests compare differences in means between 2 independent groups of numerical data (NCSS, 2016). The two independent samples are HPC levels in filtered water found at bottle refill stations and HPC levels in fountain tap water. Using NCSS, a two sample T-test was conducted.

\section{RESULTS}

Interpretation of Results. The count of the two variable groups were equal as planned with 19 samples per group. The mean number of heterotrophic bacteria was $90.74 \mathrm{cfu} / \mathrm{mL}$ in filtered water compared to the mean number of heterotrophic bacteria in tap water $86.89 \mathrm{cfu} / \mathrm{mL}$. The shows that it would be expected to find more heterotrophic bacteria in filtered water from bottle refill stations compared to regular drinking fountains.

Table 1. Heterotrophic bacteria count

\begin{tabular}{|r|r|r|r|r|r|r|}
\hline \multirow{2}{*}{$\begin{array}{r}\text { Sample } \\
\text { Location }\end{array}$} & \multicolumn{7}{|c|}{ fPC in cfu/mL } \\
\hline 1 & 106 & 0 & 39 & 4 & & \\
2 & 19 & 2 & & & & \\
3 & $0 *$ & $648^{*}$ & & & & \\
4 & 7 & 1 & & 29 & & \\
5 & 233 & 0 & 26 & 0 & & \\
6 & 314 & 209 & 205 & 756 & & \\
7 & 397 & 0 & 88 & 0 & & \\
8 & 104 & 0 & & & & \\
9 & 22 & 1 & & & & \\
10 & 70 & 0 & & & & \\
11 & 5 & 1 & 2 & 0 & & \\
12 & 41 & 0 & 8 & & 38 & 0 \\
\hline
\end{tabular}

*location considered to be an outlier

To determine whether parametric or nonparametric results were to be read the tests of assumption must be examined. Looking at the non-parametric MannWhitney results, the alternative hypothesis is examined diff $\neq 0$. As the researcher did not know which group mean would be greater, a two-sample T-test was utilized rather than a one sample T-test. The P-value obtained was 0.001643 . The shows that the difference in means is statistically significant and the null hypothesis can be rejected. This suggests there is a probability of 0.001643 that the researcher has mistakenly rejected the claim that the mean HPC of filtered water is different from the mean HPC of tap water. Since non-parametric tests do not provide power, it needs to be borrowed from the parametric tests (Aspin-Welch Unequal Variance T-test). With an $\alpha$ level of 0.05 the power for a 2 sample T-test results as 0.05048 . Typically, a power of $80 \%$ or greater would be a strong result, however at only $5 \%$ the results are not very powerful. In order to increase the power a larger sample size could be used.

Outliers. While examining the crude numbers, two samples taken from the same location (one filtered and one tap) stood out as an outlier as demonstrated in Table 1 . In all other sample locations, the tap water had significantly less HPC compared to the filtered water. At this sample location, the values are completely reversed with a recorded filtered $\mathrm{HPC}$ of $0 \mathrm{cfu} / \mathrm{mL}$ and the tap water HPC of $648 \mathrm{cfu} / \mathrm{mL}$. It is highly unlikely for the tap water to have high numbers and have the corresponding filtered water to have no bacteria. Both samples have the same source water, so if the tap water had high results the filtered water should have seen high results as well. This is 
demonstrated in sample location 6 where both tap water and filtered water have high levels of HPC. Possible reasons for this outlier include: researcher error - plates were labelled on the lid and the lids could have been switched, imputed recording error while counting plates, inaccurate labelling of samples or contamination of sample during collection.

These two samples were taken out of the input data and inferential statistics were ran subsequently to examine how the results differed. When the inferential statistics were run again, the overall results remained the same with the difference in means being statistically significant. The alternate means for filtered and tap water were $95.8 \mathrm{cfu} / \mathrm{mL}$ and $55.7 \mathrm{cfu} / \mathrm{mL}$, respectively, observing a drastic change for the tap water variable. The P-value obtained was 0.000124 with a power of 0.11891 using an alpha level at 0.05 .

Omitting the two samples resulted in an increase in mean for HPC from filtered water and decreased the mean for tap water. It also made chance of error decrease 10 -fold and doubled the power. The power at $11 \%$ still is not very high. Due to the increase in statistical significance from these results the researcher decided to submit the alternate results omitting the outlier.

\section{DISCUSSION}

This study demonstrated there was a difference in heterotrophic bacteria levels from water bottle refill stations compared to tap water at BCIT. The mean HPC for filtered water was $95 \mathrm{cfu} / \mathrm{mL}$, while the mean HPC for tap water was $55 \mathrm{cfu} / \mathrm{mL}$ (when the outlier was removed). The $p$-value was 0.000124 , therefore the null hypothesis can be rejected, proving a statistically significant difference.

Samples were collected on February $2^{\text {nd }}, 2017$, approximately 6 weeks after carbon filters were changed on December 16 ${ }^{\text {th }}, 2016$ (Pereira, 2016). It is not clear how this may have affected the results, however the schedule for changing these filters is consistent and occurs every 3 months. This puts the filters halfway through their lifetime at the time sampling happened.
While examining the results, 4 samples were taken from the same location that had extremely high numbers of HPC compared to other locations. This was the water sampled from the tap and bottle refill station in the SE6 building. As BCIT has the same water source for the entire institution, this suggests there is a problem with the distribution system in that specific building. As the HPC levels were elevated for both tap water and filtered water, it seems apparent the issue is with the incoming water, rather than the filter. Looking at the results, the filtered water was higher than the tap water for both samples from SE6, however this is not surprising because the more HPC in the tap water, the more would be expected once passed through the carbon filter. Potential reasons for these elevated counts could include multiple dead ends in the piping where water travels, or location of the sample site in regards to dead ends.

When there is insufficient water flow through a distribution system, biofilms can easily develop (Percival et al., 2000b). Residual chlorine levels are much lower leading to the proliferation of HPC. This would account for the high levels that were sampled. Another reason elevated levels could have been found were mechanical or plumbing issues not observable to the researcher. The researcher had noticed the bottle refill station had been out of order a few times over the past 6 months in SE6, however it is unknown if this impacted the results.

The initial intention of the research was to measure water quality from bottle-refill stations. During the water sampling, some of the locations with carbon filters were not bottle refill stations, but rather additional types of spouts and taps that executed a carbon filter. For example, one filter water located on a spout in the Library Staff Lounge. The researcher decided that because these alternate water fountains employed the same method of filtration and have the same risk for bacterial growth, they would be included in the study and evaluated equally.

Although Canada does not provide a Maximum Acceptable Concentration (MAC) for HPC, a similar 
ceiling value can be found in the United States. The average HPC levels recorded in this study for filtered water was $95 \mathrm{cfu} / \mathrm{mL}$, well below the MAC at 500 $\mathrm{cfu} / \mathrm{mL}$, demonstrating safe water quality sampled at BCIT.

These findings agree with previous research regarding carbon filters and increased HPC levels. It is known that carbon filters act as a food source for heterotrophic bacteria, and is demonstrated by the results of this study. It was also known that residual chlorine in tap water would be sufficient to suppress the growth of HPC in tap water. When looking at the collected values for HPC in tap water, many samples had 0 or $1 \mathrm{cfu} / \mathrm{mL}$. To the knowledge of the researcher, no studies have been performed that directly examine heterotrophic bacteria from bottle refill stations.

With regular filter replacement, these bottle refill stations provide no additional contamination risk to the consumer. They are becoming widely accepted and highly utilized by the public and should be considered in replacement of pop machines that sell bottled water. The environmental benefits from decreased uptake of purchased bottled water could have a major impact if more of these bottle refill stations were installed.

\section{Knowledge Translation}

Placement of bottle refill stations plays an important role for water quality. If stations are located near dead ends where the circulation of water is limited, it increases the chance for increased HPC. Despite HPC having no direct health impacts, it can harbour potentially harmful pathogens.

This study affirms that source water plays an important role on the effectiveness of the filter when examining HPC. As demonstrated in the SE6 building, if the residual chlorine is not being maintained, carbon filters can amplify HPC levels in drinking water.

As HPC is not considered to have any health impacts, these findings are unlikely to translate into any legislation. However, if someone in the field of public health was conducting an investigation, these stations should be considered.

\section{RECCOMENDATIONS}

Before a facility installs a bottle refill station, they should evaluate whether it is feasible. Maintenance is required for these stations along with regular changing of filters which could be quite costly in the end.

This research affirms that changing carbon filters every three months (or as per manufacturers instructions) is sufficient to maintain adequate bacteriological quality of drinking water from bottle refill stations. It also confirmed that for most of $\mathrm{BCIT}$, the residual chlorine in tap water was maintained at the time of the study and the water distribution system is functioning. Public locations and institutions that install bottler refill stations with a carbon filter should have written procedures regarding scheduled replacement of filters. Having a written schedule will remind managers of this task.

\section{LIMITATIONS}

The sample size recorded for this study was rather small at 38; 19 samples came from filtered water, and 19 samples came from tap water. Samples were all taken on the same day so it does not account for any variation in water quality. Sample size is considered the greatest limitation in this study as it has a direct impact on the power of study.

After each location had been sampled once, additional samples were taken at random to increase sample size. Some of these additional samples happened to include the one area that had much higher bacterial counts than other locations. If this study were to be repeated, it cannot be guaranteed that this site would be sampled twice.

Due to budget restraints, the methods demonstrated could not precisely follow the standard methods (APHA/AWWA/WEF, 2005). This could impact the validity of the results. As well, no serial dilutions were performed to determine a 
countable plate due to resource allocation. The study had to be completed within three months.

Samples were only taken at BCIT, which has the same water source. The results can only be generalizable to all of BCIT and no other locations. The results rely on the distribution system, and the quality of source water. Other areas of the Lower Mainland in BC have the same source water however the distribution system is different everywhere.

\section{FUTURE RESEARCH}

As bottle refill stations are still near the beginning of their popularity, the opportunity for future research is immense. Studies could be conducted similar to this one performing chemical testing on water from bottle refill stations examining levels of lead, or bacteriological sampling examining aerobic colony count from bottle refill stations. Additionally, the long-term effectiveness of filters could be examined - testing HPC at different time intervals ie. week 0 , week 1 , week 2 etc). A survey could also be conducted regarding usage and acceptability of bottle refill stations in public locations.

\section{CONCLUSION}

Despite not having any direct health effects, having heterotrophic bacteria in a water supply is not desirable. No MAC is set out by Canadian guidelines, however it would be unacceptable to see more than $500 \mathrm{cfu} / \mathrm{ml}$ in a sample and could interfere with total coliform count (Federal-Provincial-Territorial Committee on Drinking Water, 2014). Biofilms can develop in a distribution system, potentially harboring pathogenic bacteria. It is also common to see a biofilm develop in water coolers as well as point of use water treatment (Chaidez \& Gerba, 2004). Proper cleaning and replacement of filters should be seen to reduce risk of contamination.

Heterotrophs are generally not believed to be the cause of disease in humans but their presence may give an indication that control within a distribution system is not satisfactory (Amanidaz, Zafarzadeh, \& Mahvi, 2015). Colony counts are only useful as a parameter if they are used continuously to monitor a system. A sudden increase over the numbers normally found in a system or a sustained increase over a period is suggestive of a failure in disinfection or colonization of the system, respectively.

Bottle refill stations are well constructed to provide safe and enjoyable drinking water to the public. With proper training and maintenance by operators, there is no need for concern. If these stations are neglected, contamination could occur, potentially making members of the public ill. With an increase in the number of these stations, we could likely see an increase in gastrointestinal illness.

This study confirmed that bottle refill stations that utilize a carbon filter to filter drinking water have different levels of heterotrophic bacteria compared to tap water. At BCIT where sampling took place, filters are changed every three months. This time frame between filter replacement appears to be sufficient as HPC levels were well below $500 \mathrm{cfu} / \mathrm{mL}$ for the majority of sampling locations. One location (in building SE6) that recorded high levels of HPC gave the impression of having lower water quality compared to the rest of the campus. This study needs to be repeated to confirm results.

\section{ACKNOWLEDGEMENTS}

The author would like to acknowledge her colleagues of the Environmental Health class of 2017, and Dr. Helen Heacock for all their extended support. The author would also like to thank Janek Kobylarz for his involvement in the study.

\section{COMPETING INTERESTS}

The authors declare that they have no competing interests.

\section{REFERENCES}

Amanidaz, N., Zafarzadeh, A., \& Mahvi, A. H. (2015). The Interaction between Heterotrophic Bacteria and Coliform, Fecal 
Coliform, Fecal Streptococci Bacteria in the Water Supply Networks. Iran J Public Health, 44(12), 1685-1692. Retrieved from http://ijph.tums.ac.ir

APHA/AWWA/WEF. (2005). Standard Methods for the Examination of Water and Wastewater. American Public Health Association, American Water Works Association, and Water Environment Federation.

Association, A. P. H. (1999). Standard Methods for the Examination of Water and Wastewater. American Public Heatlh Association American Water Works Association Water Environment Association.

Chaidez, C., \& Gerba, C. P. (2004). Comparison of the microbiologic quality of point- of-use (POU)-treated water and tap water. International Journal of Environmental Health Research, 14(4), 253-60.

https://doi.org/10.1080/09603120410001725 595

Elkay. (2017). ELKAY | Elkay Unveils \&quot;Next Generation\&quot; Bottle Filling Station. Retrieved January 19, 2017, from http://www.elkay.com/news-bottle-fillingstation

ELKAY | Elkay EZH2O Bottle Filling Station with Single Cooler Filtered, 8 GPH Vandal-Resistant Stainless LVRC8WSK. (n.d.). Retrieved from http://www.elkay.com/lvrc8wsk

Epa, U. (2009). National Drinking Water

Regulations MCL Booklet May 2009. Retrieved October 22, 2016, from

https://www.epa.gov/sites/production/files/2 016-

06/documents/npwdr_complete_table.pdf

Federal-Provincial-Territorial Committee on Drinking Water. (2014). Guidelines for Canadian Drinking Water Quality Summary Table. Retrieved October 15, 2016, from http://www.hc-sc.gc.ca/ewhsemt/alt_formats/pdf/pubs/watereau/sum_guide-res_recom/sum_guideres_recom_2014-10_eng.pdf

Health Canada. (2012). Guidance on the Use of Heterotrophic Plate Counts in Canadian Drinking Water Supplies. Water. Air and Climate Change Bureau, Healthy
Environments and Consumer Safety Branch, Health Canada, Ottawa, Ontario. Retrieved from

http://healthycanadians.gc.ca/publications/h ealthy-living-vie-saine/water-heterotrophicheterotrophes-eau/alt/water-heterotrophicheterotrophes-eau-eng.pdf

Levesque, B., Simard, P., Gauvin, D., Gingras, S., Dewailly, E., \& Letarte2, R. (1994).

Comparison of the Microbiological Quality of Water Coolers and That of Municipal Water Systems. APPLIED AND ENVIRONMENTAL MICROBIOLOGY, 6O(4), 1174-1178.

Metro Vancouver. (2016). Drinking Water Facilities. Retrieved January 19, 2017, from http://www.metrovancouver.org/services/wa ter/quality-facilities/facilitiesprocesses/drinking-water-treatmentfacilities/Pages/default.aspx

NCSS. (2016). No Title.

Neogen. (2009). R2A Agar.

Oasis, F. by. (n.d.). OUR DRINKING FOUNTAINS WHEN EQUIPPED WITH THE VERSAFILTER FILTRATION SYSTEM. Retrieved October 23, 2016, from http://www.franke.com/content/dam/comm ercial_systems/ca/en/service_and_support/lit erature_and_videos/General Information/Versafilter_Flyer-LR.pdf

Percival, S. L., Walker, J. T., \& Hunter, P. R. (2000a). Biofilm Development in General. In Microbiological Aspects of Biofilms and Drinking Water (pp. 61-84). CRC Press. https://doi.org/doi:10.1201/9781420041941. ch6

Percival, S. L., Walker, J. T., \& Hunter, P. R. (2000b). Biofilm Formation in Potable Water. In Microbiological Aspects of Biofilms and Drinking Water (pp. 85-102). CRC Press. https://doi.org/doi:10.1201/9781420041941. ch7

Percival, S. L., Walker, J. T., \& Hunter, P. R. (2000c). Microbes and Public Health Significance. In Microbiological Aspects of Biofilms and Drinking Water (pp. 103-154). CRC Press. https://doi.org/doi:10.1201/9781420041941. ch8

Pereira, J. (2016). Drinking Water Fountains. 
Burnaby.

Schillinger, J., \& S, D. V. K. (2004). Drinking-water quality and issues associated with water vending machines in the city of Los Angeles. Journal of Environmental Health, 66(6), 2546. Retrieved from http://proxy.lib.sfu.ca/login?url=http://search .ebscohost.com/login.aspx?direct=true $\& \mathrm{db}=\mathrm{c}$ cm\&AN=106576961\&site=ehost-live 